\section{SAT0014 DECREASED CIRCULATING CD19 +CD24HICD38HIREGULATORY B CELLS IN ACPA POSITIVE RHEUMATOID ARTHRITIS: EFFECT OF IL-6 RECEPTOR BLOCKADE}

Paula Fortea-Gordo ${ }^{1}$, Alejandro Villalva ${ }^{1}$, Laura Nuño ${ }^{1}$, Maria-Jose SantosBornez $^{1}$, Irene Monjo ${ }^{1}$, Diana Peiteado ${ }^{1}$, Amaya Puig-Kröger ${ }^{2}$, Paloma SanchezMateos $^{2}$, Alejandro Balsa', Maria-Eugenia Miranda-Carus ${ }^{1} .{ }^{1}$ Hospital La PazIdiPAZ, Rheumatology, Madrid, Spain; ${ }^{2}$ Gregorio Marañón Hospital, ImmunoOncology, Madrid, Spain

Background: $C D 19+C D 24^{\text {hi }} C D 38^{\text {hi }} B$ cells have a regulatory capacity and their frequency is altered in the peripheral blood of patients with various autoimmune conditions, including RA.

Objectives: To determine the frequency of circulating $\mathrm{CD} 19+\mathrm{CD} 24^{\mathrm{hi}} \mathrm{CD} 38^{\mathrm{hi}}$ regulatory $B$ cells (cBreg) in ACPA+ vs ACPA- patients with RA, and its possible modification by IL-6R blockade.

Methods: Peripheral blood was drawn from ACPA+ $(n=37)$ or ACPA$(n=19) \quad R A$ patients treated with conventional synthetic DMARDs (csDMARDs), and from ACPA+ $(n=31)$ or ACPA- $(n=12)$ RA patients treated with tocilizumab; in additon one age and gender-matched healthy control was studied alongside with each patient $(n=99)$. After isolation by Ficoll-Hypaque gradient, PBMCs were stained with antibodies to CD3, CD4, CD19, CD24, and CD38, and examined by flow cytometry.

Results: A decreased frequency of cBreg cells was observed in ACPA+ but not ACPA- RA patients treated with csDMARDs. Interestingly, the frequency of cBreg cells showed a significant negative correlation with ACPA titers $(r=-0.44, p=0.021)$. In contrast, no correlation was found between the frequency of cBreg cells and either RF titres or disease activity as determined by the DAS28 score. However, not only in ACPAbut also in ACPA+ patients receiving tocilizumab, the percentage of cBreg cells was not different from that observed in HC. Four ACPA+ patients treated with csDMARDs who initiated tocilizumab, demonstrated a significant elevation of their cBreg frequency at 12 months.

Conclusion: A decreased frequency of cBreg cells is apparent in ACPA+ but not ACPA- RA patients receiving csDMARDs, that is related with ACPA titres but not with RF titres or disease activity. In contrast, in $\mathrm{ACPA}+$ patients treated with tocilizumab, the frequency of CD19 $+\mathrm{CD} 24^{\mathrm{hi}} \mathrm{CD} 38^{\mathrm{hi}} \mathrm{B}$ cells is comparable to that observed in $\mathrm{HC}$, suggesting that IL-6R blockade is able to modify the altered Breg cell balance.

\section{REFERENCE}

[1] Flores-Borja F, et al. Sci Transl Med. 2013;5:173ra23.

Disclosure of Interests: Paula Fortea-Gordo: None declared, Alejandro Villalva: None declared, Laura Nuño: None declared, Maria-Jose Santos-Bornez: None declared, Irene Monjo: None declared, Diana Peiteado: None declared, Amaya Puig-Kröger: None declared, Paloma Sanchez-Mateos: None declared, Alejandro Balsa Grant/research support from: Abbvie, Pfizer, Novartis, BMS, Nordic, Sanofi, Consultant for: Abbvie, Pfizer, Novartis, BMS, Nordic, Sanofi, Sandoz, Lilly, Paid instructor for: Pfizer, Speakers bureau: Pfizer, Novartis, UCB, Nordic, Sanofi, Sandoz, Lilly, Maria-Eugenia Miranda-Carus Grant/research support from: Roche Pharma, BMS

DOI: 10.1136/annrheumdis-2019-eular.3213

\section{SAT0015 TREATMENT WITH ABATACEPT BUT NOT WITH TNF BLOCKERS, IS ASSOCIATED WITH A REDUCTION OF CONSTITUTIVELY ELEVATED CIRCULATING FOLLICULAR HELPER T CELLS IN RHEUMATOID ARTHRITIS}

Paula Fortea-Gordo, Laura Nuño, Alejandro Villalva, Maria-Jose Santos-Bornez, Diana Peiteado, Irene Monjo, Alejandro Balsa, Maria-Eugenia Miranda-Carus. Hospital La Paz - IdiPAZ, Madrid, Spain

Background: Circulating CD4 T cells that express CXCR5 together with PD-1 and/or ICOS are considered as counterparts of bona fide Th cells and function as $\mathrm{B}$ cell helpers. In addition, circulating CD4+CXCR5+ T cells can be subdivided into three subpopulations: CXCR5+CXCR3+CCR6(Tfh-Th1), CXCR5+CXCR3-CCR6+ (Tfh-Th17) and CXCR5+CXCR3-CCR6(Tfh-Th2). Only Thh-Th17 and Tfh-Th2 but not Thf-Th1 cells seem to provide B cell help. Altered frequencies of circulating Tfh cells (cTfh) and of cTfh cell subpopulations have been associated with autoimmune conditions. We previously described that patients with RA treated with conventional synthetic DMARDs (csDMARDs), demonstrate constitutively altered frequencies of cTfh and cTfh cell subpopulations that are observed not only in patients with an active disease but also in patients who are in remission.

Objectives: To determine if treatment with biological agents (TNF blockers or abatacept) is able to modify the constitutively altered cTfh and cTfh subpopulation numbers, observed in RA patients receiving csDMARDs Methods: Peripheral blood was drawn from RA patients receiving csDMARDS $(n=22)$, TNF blockers $(n=21: 10$ infliximab, 6 etanercept, 3 certolizumab, 2 adalimumab), or abatacept $(n=17)$. For each patient, an age and gender-matched healthy control was also studied $(n=60)$. cTfh and plasmablast frequencies were determined by flow cytometry of freshly isolated PBMCs.

Results: As described, RA patients receiving csDMARDs demonstrated whether they had an active or inactive disease, an increased frequency

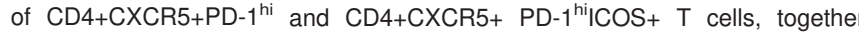
with an increased frequency of circulating plasmablasts. In addition, the frequency of Tfh-Th1 cells was significantly decreased and the frequency of Tfh-Th17 and Tfh-Th2 cells were significantly increased as compared with HC; subsequently, the ratio (Tfh-Th17+Tfh-Th2)/Thh-Th1 was increased in RA: that is, RA patients demonstrated a higher proportion of Tfh cell subsets bearing a phenotype associated with B cell helping capacity. Interestingly, these alterations were also observed in RA patients treated with TNF blockers, whether they had an active or inactive disease. In contrast, in patients receiving abatacept, the frequencies of cTfh, cTfh cell subpopulations and plasmablasts, were not different from those observed in $\mathrm{HC}$

Conclusion: Patients with RA receiving csDMARDs or TNF blockers demonstrated a constitutively increased frequency of cTh cells and an overrepresentation of CTfh subsets bearing a B cell helper phenotype suggesting that altered germinal center dynamics play a role in RA pathogenesis. Remarkably, in RA patients treated with abatacept these frequencies and ratio were not altered, indicating that costimulation blockade is able to revert the increased generation of Tfh cells in RA; conversely, TNF neutralization does not seem to affect the generation or recirculation of cTfh.

\section{REFERENCE}

[1] Simpson N et al, Arthritis Rheum 2010; Craft J, Nat Rev Rheumatol 2012; Morita R, et al., Immunity 2011; Arroyo-Villa I, et al., Arthritis Res Ther 2014.

Disclosure of Interests: Paula Fortea-Gordo: None declared, Laura Nuño: None declared, Alejandro Villalva: None declared, Maria-Jose Santos-Bornez: None declared, Diana Peiteado: None declared, Irene Monjo: None declared, Alejandro Balsa Grant/research support from: Abbvie, Pfizer, Novartis, BMS, Nordic, Sanofi, Consultant for: Abbvie, Pfizer, Novartis, BMS, Nordic, Sanofi, Sandoz, Lilly, Paid instructor for: Pfizer, Speakers bureau: Pfizer, Novartis, UCB, Nordic, Sanofi, Sandoz, Lilly, Maria-Eugenia Miranda-Carus Grant/research support from: Roche Pharma, BMS DOI: 10.1136/annrheumdis-2019-eular.3287

\section{SAT0016 RHEUMATOID ARTHRITIS PATIENTS DISPLAY B-CELL DYSREGULATION ALREADY IN THE NAÏVE REPERTOIRE}

Yan Wang ${ }^{1}$, Katy A. Lloyd ${ }^{1}$, loannis Melas ${ }^{2}$, Daniel Ramsköld ${ }^{1}$, Diana Zhou ${ }^{1}$, Radha Thyagarajan ${ }^{1}$, Karin Lundberg ${ }^{1}$, Lars Klareskog ${ }^{1}$, Anca Catrina ${ }^{1}$, Stephen Rapecki ${ }^{2}$, Vivianne Malmström ${ }^{1}$, Caroline Grönwall'. ${ }^{1}$ Karolinska Institutet, Division of Rheumatology, Department of Medicine, Stockholm, Sweden; ${ }^{2}$ UCB Pharma, Slough, United Kingdom

Background: Seropositive rheumatoid arthritis (RA) is associated with autoreactivity to citrullinated proteins and rheumatoid factor activity. The adaptive immune system and $B$ cells are postulated to be central in RA pathogenesis, yet possible B-cell dysregulations have not been extensively studied. Here, we use exploratory mass cytometry and next generation sequencing to study overall $B$ cell repertoire shifts in RA patients compared to healthy individuals.

Objectives: We hypothesized that there are an underlying B cells repertoire distortion in RA that may be an important key to understanding the autoimmune pathogenesis.

Methods: B cells enriched from PBMCs from nine ACPA pos., seven ACPA neg. RA patients, and six matched heathy controls were phenotyped with mass cytometry (CyTOF) using a 35-marker panel. Single cell data from all samples were pooled clustered via flowSOM in R to identify distinct cell populations, and visualized with tSNE. Cell numbers in each cluster/population were calculated to identify over-representation in patient groups or controls. ANOVA was performed to account for confounding 
factors such as age, sex and cell preparation date and differential expression of markers between groups was calculated via t-test. In parallel, PBMC B cell receptor (BCR) repertoires were investigated in 13 ACPA pos. RA patients and six age-matched healthy donors using the Illumina MiSeq platform and PCR multiplex amplicon libraries with a molecular barcode strategy to generate full variable region coverage. Sequences were filtered using PRESTO, annotated by IMGT and Change-O, finally generating 587000 unique V-regions. Total serum IgM levels were screened by sandwich ELISA in 157 population controls, 193 ACPA pos., and 50 ACPA neg. RA patients. Variable gene frequency was analyzed by Chi-square with Yates correction and serum IgM levels with Kruskal-Wallis test

Results: Several B-cell phenotypes were found to be significantly different in ACPA pos. RA compared to controls including an increase in HLA-DR across subsets, $C D 11 \mathrm{c}$ in $\lg \mathrm{A}$ memory and $\mathrm{CD} 22$ expression in clusters of mature naïve IgM positive $B$ cells. Moreover, we could see lower circulating cell counts in ACPA pos. $R A$ in $\lg$ memory $(p=0.01)$ and trends for elevation in an CXCR5/CCR6 high transitional B cell cluster $(p=0.06)$, with parallel lower number of transitional $B$ cells with lower CCR6 expression $(p=0.06)$. Notably, ACPA neg. RA generally had an intermediate phenotype between healthy controls and ACPA pos. RA. Several significant shifts in the RA BCR repertoire could be observed, including an expected higher frequency of $\mathrm{VH} \mathrm{N}$-linked glycosylation in highly mutated BCR $(p<0.0001)$. Yet, the most striking difference was a significantly higher frequency of $\mathrm{VH}$ with low somatic hypermutation (SHM) levels in RA-derived B cells ( $<5$ mutations, $p<0.000114 .7 \%$ vs $8,7)$. This was seen in all sequences, both $\operatorname{lgM}$ and class-switched, but was especially prominent in lgG1 rearrangements $(9.6 \%$ vs $18.8 \%$ low mutation, $\mathrm{p}<0.0001 \mathrm{OR}=2.2 \mathrm{Cl}: 2.0-2.35)$. In line with an $\operatorname{lgM}$ and low mutation profile in RA, we also observed that both ACPA pos. $(p<0.0001)$ and ACPA neg. $(p<0.001)$ RA patients have a significant increase in total $\operatorname{lgM}$ levels compared to controls $(1.4 \pm 0.7 ; 1.3 \pm$ $0.7 ; 1.0 \pm 0.6 \mathrm{mg} / \mathrm{ml}$, respectively).

Conclusion: Previous studies have shown that anti-citrulline autoreactivity in RA is primarily originates from memory $B$ cells and characterized by high somatic mutations and N-glycosylation sites. However, here the largest $B$ cell distortions in ACPA positive RA are observed in the naïve B cell population that have not undergone germinal center responses. These differences could reflect baseline shifts and elevated natural autoreactivity as an underlying mechanism in RA pathogenesis.

Disclosure of Interests: Yan Wang: None declared, Katy A. Lloyd: None declared, Ioannis Melas Employee of: Employed by UCB Pharma, Daniel Ramsköld: None declared, Diana Zhou: None declared, Radha Thyagarajan: None declared, Karin Lundberg: None declared, Lars Klareskog Grant/research support from: Yes, but not for the presented study., Anca Catrina Grant/research support from: Yes, but not for the presented study., Stephen Rapecki Employee of: Employed by UCB Pharma, Vivianne Malmström: None declared, Caroline Grönwall: None declared DOI: 10.1136/annrheumdis-2019-eular.7285

\section{SAT0017 COMPARISON OF IMMUNOREGULATORY WITH CONVENTIONAL DMARDS THERAPY IN PATIENTS WITH RHEUMATOID ARTHRITIS}

Qiao ling Guo ${ }^{1}$, LI Bao Chen ${ }^{1}$, LI Zhao-Hua ${ }^{1}$, Yan Yan Wang ${ }^{1}$, Xin Wang ${ }^{1}$, Chong Gao ${ }^{2}$, LI Xiaofeng ${ }^{1}$, Caihong Wang ${ }^{1} .{ }^{1}$ The second hospital of Shanxi Medical University, Rheumatology Department, Taiyuan, China; ${ }^{2}$ Department of Pathology, Brigham and Women's Hospital, Harvard Medical School, Boston, MA, USA., Department of Pathology, Massachusetts, American Samoa

Background: Rheumatoid arthritis (RA) is a chronic systemic autoimmune disease characterized by joint synovitis that ultimately leads to joint destruction and loss of function. Conventional therapy or standard medical treatment of RA involves the disease-modifying antirheumatic drugs (DMARDs). Because DMARDs act nonspecifically on the patient's immune cells, it can weaken the body's ability to fight infections. We previously reported that imbalance of effector and regulatory $T$ cells (Tregs) that should be an important cause of RA. Therefore, we developed an immunomodulatory therapy for restoring the reduced Treg cells is the main direction for the treatment of RA. [1]

Objectives: To compare the effects of new immunoregulatory therapy and conventional DMARDs therapy on disease remission and immune homeostasis in RA patients, and to investigate the efficacy of immunoregulatory therapy in the reconstruction of immune tolerance.

Methods: The study included 184 patients with RA (meeting the diagnostic classification criteria for RA revised by ACR in 1987).According to the therapeutic regimen they have received, there were assigned to the immunoregulatory (144 patients, Sirolimus capsule, $0.5 \mathrm{mg}$ twice a week and Tretinoin tablets, $10 \mathrm{mg}$ once a day, for 12 weeks) and conventional DMARDs group (40 cases, Leflunomide tablets, $10 \mathrm{mg}$ once a day for 12 weeks). Other drugs are similar between the two groups. The absolute numbers of Th17 and Treg cells in peripheral blood were measured by Flow Cytometer (FCM).

Results: 1. There was no statistically significant difference in DAS28 score $(P>0.05)$ between the two groups. 2 . In the immunomodulatory group, there were statistical differences in the number of Treg as compared with normal controls $(P<0.05)$. After immunoregulatory therapy, the absolute number of Treg cells was increased, leading to ratio of Th17/ Treg decreased (Table1, Figure1). 3. Conventional DMARDs therapy reduced, significantly the number of Treg cells $(P<0.05)$, leading to a increase in the ratio of Th17/Tregs (Table 1, Figure 2).

Conclusion: The results of this study show that the remission rates of immunomodulation group and conventional DMARDS group were $37.39 \%$ and $36.00 \%$, respectively. There was no significant difference between the two groups. But as for the effects on their immune cells, immunomodulatory therapy can increase the level of Treg cells compared with conventional DMARDs. It also shows that immunomodulatory therapy may play an important role in restoring immune tolerance in patients with RA, which may help us find new ideas for treating RA. However we need to enlarge the sample size and extend the treatment time to observe the long-term efficacy of the immunomodulatory therapy in the population.

\section{REFERENCE}

[1] Benson R A, Patakas A, Mcqueenie R, et al. Arthritis in space and time To boldly go![J]. Febs Letters, 2011, 585(23):3640-3648.
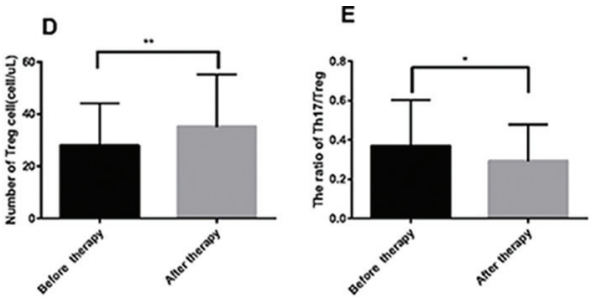

Figure 1. The number of Treg cells and the ratio of Th17/Treg before and after immunomodulatory therapy.

Table 1. The number of Th17 and Treg cell (cell/ul), ratio of Th17/Treg in immunomodulatory therapy and Traditional DMARDs therapy.

\begin{tabular}{|c|c|c|c|c|c|c|}
\hline \multirow{2}{*}{$\begin{array}{l}\text { Immune } \\
\text { Cells }\end{array}$} & \multicolumn{2}{|c|}{ Immunomodulatory $\mathrm{n}=144$} & \multicolumn{2}{|c|}{ Traditional DMARDs $\mathrm{n}=40$} & \multirow{2}{*}{$\begin{array}{c}P \text { value } A \text { vs } \\
B\end{array}$} & \multirow{2}{*}{$\begin{array}{c}P \text { value } C \text { vs } \\
D\end{array}$} \\
\hline & $\begin{array}{l}\text { Before therapy } \\
\text { (A) }\end{array}$ & $\begin{array}{l}\text { After therapy } \\
\text { (B) }\end{array}$ & $\begin{array}{l}\text { Before therapy } \\
\text { (C) }\end{array}$ & $\begin{array}{c}\text { After therapy } \\
\text { (D) }\end{array}$ & & \\
\hline Th17 & $\begin{array}{c}8.6453 \\
(4.673,11.000)\end{array}$ & $\begin{array}{c}9.2359 \\
(4.440,12.570)\end{array}$ & $\begin{array}{c}7.4693 \\
(4.300,10.850)\end{array}$ & $\begin{array}{c}9.8622 \\
(4.045,8.100)\end{array}$ & 0.086 & 0.512 \\
\hline Treg & $\begin{array}{c}27.9202 \\
(15.900,36.270)\end{array}$ & $\begin{array}{c}31.9208 \\
(22.90,39.7775)\end{array}$ & $\begin{array}{c}38.6240 \\
(31.190,43.120)\end{array}$ & $\begin{array}{c}30.2330 \\
(24.150,37.380)\end{array}$ & 0.021 & 0.001 \\
\hline Th17/Treg & $\begin{array}{c}0.3669 \\
(0.199,0.490)\end{array}$ & $\begin{array}{c}0.2907 \\
(0.172,0.341)\end{array}$ & $\begin{array}{c}0.2343 \\
(0.127,0.347)\end{array}$ & $\begin{array}{c}0.3581 \\
(0.202,0.515)\end{array}$ & 0.020 & 0.024 \\
\hline
\end{tabular}

